\title{
Fuzzy fractional Ostrowski inequality with Caputo differentiability
}

\author{
Tofigh Allahviranloo' ${ }^{1}$ Lutfi Avazpour ${ }^{2 *}$, Mohammad J Ebadi ${ }^{3}$, Dumitru Baleanu ${ }^{4,5,6}$ and Soheil Salahshour ${ }^{7}$
}

A retraction article was published for this article. It is available from the following link; http://www.journalofinequalitiesandapplications.com/content/2013/1/417.

"Correspondence:

avazpour.l@gmail.com

${ }^{2}$ Department of Mathematics,

Yasooj Branch, Islamic Azad

University, Yasooj, Iran

Full list of author information is

available at the end of the article

\begin{abstract}
The use of fractional inequalities in mathematical models is increasingly widespread in recent years. In this manuscript, we firstly propose the right Caputo derivative of fuzzy-valued functions about fractional order $v(0<v<1)$. To this end, we consider two types of differentiability (similar to the non-fractional case). Then we derive the equivalent integral forms of original fuzzy fractional differential equations. Finally, we prove the fuzzy Ostrowski inequality involving three functions under Caputo's differentiability. In this regard, we state some new results.
\end{abstract}

Keywords: fuzzy fractional Ostrowski inequality; fuzzy Caputo differentiability; Hukuhara difference; fuzzy-valued function

\section{Introduction}

In 1938, Ostrowski proved the following important inequality [1]:

Theorem 1.1 Let $f:[a, b] \rightarrow \mathbb{R}$ be continuous on $[a, b]$ and differentiable on $(a, b)$ whose derivative $f^{\prime}:(a, b) \rightarrow \mathbb{R}$ is bounded on $(a, b)$, i.e., $\left|f^{\prime} \|_{\infty}=\sup _{t \in(a, b)}\right| f^{\prime}(t) \mid<\infty$. Then

$$
\left|\frac{1}{b-a} \int_{a}^{b} f(t) d t-f(x)\right| \leq\left[\frac{1}{4}+\frac{\left(x-\frac{a+b}{2}\right)^{2}}{(b-a)^{2}}\right](b-a)\left\|f^{\prime}\right\|_{\infty}
$$

for any $x \in[a, b]$.

The constant $\frac{1}{4}$ is best possible in the sense that it cannot be replaced by a smaller one. This inequality gives an upper bound for the approximation of the integral average $\frac{1}{b-a} \int_{a}^{b} f(t) d t$ by the value $f$ at a point $x \in(a, b)$. We notice that the first generalization of Ostrowski's inequality was given by Milovanovic and Pecaric in [2].

In recent years, these inequalities have been studied by many researchers, and numerous generalizations, extensions and variants of them have been considered in a number of papers (see Refs. [3-10] and the references therein). In this way, some new types of inequalities such as inequalities of Ostrowski-Gruss type, inequalities of Ostrowski-Chebyshev type, etc. were formed. The first inequality of Ostrowski-Gruss type was given by Dragomir and Wang in [11], and it was generalized and improved by Matic, Pecaric and Ujevic in [12]. Also, Cheng gave a sharp version of the mentioned inequality in [13]. 
In $[3,14]$ Pachpatte has proved the Ostrowski inequality in three independent variables. In the past few years, many authors have obtained various generalizations of this type of inequality and many researchers worked on a fractional form of it as well as on time scale calculus (see, for example, Refs. [15-18] and the references therein).

We mention that these inequalities were applied for Euler's beta mapping and special means such as the arithmetic mean, the geometric mean, the harmonic mean and so on (for more details, see Ref. [19] and the references therein). In [20] the authors have applied this inequality for the error bounds of general Riemman's quadrature formulae in terms of $\left\|f^{\prime}\right\|_{1}$.

The main purpose of this manuscript is to establish Ostrowski-type inequality involving Caputo differentiability. So, we propose the right fuzzy Caputo derivative and the fuzzy right fractional Taylor formula in order to prove Ostrowski's inequality. Then we use these concepts to prove this inequality involving three functions. Some more details about fuzzy differential equations and their applications can be found in Refs. [21-24] and the references therein.

This manuscript is organized as follows. In Section 2, we recall some basic concepts. In Section 3, we firstly propose the right Caputo derivative in the sense of $[(i)-v]$ differentiability and $[(i i)-v]$-differentiability, then the equivalent integral form of the original fuzzy fractional differential equation is obtained. After that, we prove the fuzzy Ostrowski inequality involving three functions.

\section{Basic concepts}

We denote the set of all real numbers by $\mathbb{R}$, and the set of all fuzzy numbers on $\mathbb{R}$ is indicated by $R_{F}$. A fuzzy number is a mapping $u: \mathbb{R} \rightarrow[0,1]$ with the following properties:

(a) $u$ is upper semi-continuous,

(b) $u$ is fuzzy convex, i.e., $u(\lambda x+(1-\lambda) y) \geq \min \{u(x), u(y)\}$ for all $x, y \in \mathbb{R}, \lambda \in[0,1]$,

(c) $u$ is normal, i.e., $\exists x_{0} \in \mathbb{R}$ for which $u\left(x_{0}\right)=1$,

(d) $\operatorname{supp} u=\{x \in \mathbb{R} \mid u(x)>0\}$ is the support of the $u$, and its closure $\operatorname{cl}(\operatorname{supp} u)$ is compact.

For $u, v \in R_{F}$ and $\lambda \in \mathbb{R}$, we define uniquely the sum $u \oplus v$ and the product $\lambda \odot u$ by

$$
[u \oplus v]^{r}=[u]^{r}+[v]^{r}, \quad[\lambda \odot u]^{r}=\lambda[u]^{r}, \quad \forall r \in[0,1]
$$

where $[u]^{r}+[v]^{r}$ means the usual addition of two integrals (as subsets of $\mathbb{R}$ ) and $\lambda[u]^{r}$ means the usual product between a scalar and a subset of $\mathbb{R}$.

Define $D: R_{F} \times R_{F} \longrightarrow \mathbb{R}_{+}$by

$$
D(u, v):=\sup _{r \in[0,1]} \max \left\{\left|u_{-}(r)-v_{-}(r)\right|,\left|u_{+}(r)-v_{+}(r)\right|\right\},
$$

where

$$
[v]^{r}=\left[v_{-}(r), v_{+}(r)\right], \quad u, v \in R_{F} .
$$

We have that $D$ is a metric on $R_{F}$. Then $\left(R_{F}, D\right)$ is a complete metric space with the following properties:

$D(u \oplus w, v \oplus w)=D(u, v), \forall u, v, w \in R_{F}$, 
$D(k \odot u, k \odot v)=|k| D(u, v), \forall u, v \in R_{F}, \forall k \in \mathbb{R}$,

$D(u \oplus v, w \oplus e)=D(u, w)+D(v, e), \forall u, v, w, e \in R_{F}$.

Let us consider $x, y \in R_{F}$. If there exists $z \in R_{F}: x=y \oplus z$, then we call $z$ the H-difference on $x$ and $y$, denoted by $x \ominus y$.

Let us consider $f:[a, b] \rightarrow R_{F}$. We say that $f$ is fuzzy Riemann integrable to $I \in R_{F}$ if for any $\epsilon>0$, there exists $\delta>0$ such that for any division $P=\{[u, v] ; \xi\}$ of $[a, b]$ with the norms $\Delta(P)<\delta$, we have

$$
D\left(\sum_{P}^{*}(v-u) \odot f(\xi), I\right)<\epsilon,
$$

and we write

$$
I:=(F R) \int_{a}^{b} f(x) d x
$$

Let $f:[a, b] \rightarrow R_{F}$ be fuzzy continuous. Then $(F R) \int_{a}^{b} f(x) d x$ exists and belongs to $R_{F}$; furthermore, it holds $\left[(F R) \int_{a}^{b} f(x) d x\right]^{r}=\left[\int_{a}^{b}(f)_{-}(r)(x) d x,(f)_{+}(r)(x) d x\right], \forall r \in[0,1]$.

We consider $f \in C_{F}([a, b])$ and $c \in[a, b]$. Then $(F R) \int_{a}^{b} f(x) d x=(F R) \int_{a}^{c} f(x) d x+$ (FR) $\int_{c}^{b} f(x) d x$.

Let $f, g \in C_{F}([a, b])$ and $c_{1}, c_{2} \in[a, b]$. Then $(F R) \int_{a}^{b}\left(c_{1} f(x)+c_{2} g(x)\right) d x=c_{1}(F R) \int_{a}^{b} f(x) d x+$ $c_{2}(F R) \int_{a}^{b} g(x) d x$. Also, if $f, g:[a, b] \subseteq R \rightarrow R_{F}$ are fuzzy continuous functions, then the function $F:[a, b] \rightarrow \mathbb{R}_{+}$defined by $F(x):=D(f(x), g(x))$ is continuous on $[a, b]$, and

$$
\begin{aligned}
& D\left((F R) \int_{a}^{b} f(x) d x,(F R) \int_{a}^{b} g(x) d x\right) \\
& \quad \leq \int_{a}^{b} D(f(x), g(x)) d x .
\end{aligned}
$$

Let $f \in C_{F}([a, b]), 0<v<1$. Then we define the fuzzy fractional left Riemann-Liouville operator as

$$
I_{a+}^{v} f(x)=\frac{1}{\Gamma(v)} \odot \int_{a}^{x}(x-t)^{v-1} \odot f(t) d t, \quad x \in[a, b] .
$$

Also, we define the fuzzy fractional right Riemann-Liouville operator by

$$
I_{b-}^{v} f(x)=\frac{1}{\Gamma(v)} \odot \int_{x}^{b}(t-x)^{v-1} \odot f(t) d t, \quad x \in[a, b] .
$$

Above, $\Gamma$ denotes the gamma function

$$
\Gamma(v)=\int_{0}^{\infty} e^{-t} t^{\nu-1} d t
$$

\section{Main results}

In this section, we state some definitions and results about the right fuzzy Caputo derivative and the fuzzy right fractional Taylor formula in order to prove the Ostrowski inequality involving three functions. 
Definition 3.1 Let $f \in C_{F}[a, b] \cap L_{F}[a, b], f^{\prime}$ be integrable. Then the right fuzzy Caputo derivative off for $0<v<1$ and $x \in[a, b]$ is denoted by $D_{b-}^{v} f(x) \in R_{F}$ and defined by

$$
D_{b-}^{v} f(x)=\frac{(-1)}{\Gamma(1-v)} \odot \int_{x}^{b}(t-x)^{-v} \odot f^{\prime}(t) d t .
$$

Now, we state an efficient result.

Theorem 3.1 Let $f \in C_{F}[a, b] \cap L_{F}[a, b], 0<v<1, a \leq x \leq b$.

(i) Let $f$ be (ii)-differentiable, then we have $[(i)-v]$-differentiable right fuzzy Caputo derivative and

$$
\left(D_{b-}^{v} f\right)(x, r)=\left[\left(D_{b^{-}}^{v} f_{-}\right)(x, r),\left(D_{b^{-}}^{v} f_{+}\right)(x, r)\right], \quad 0 \leq r \leq 1 .
$$

(ii) Let $f$ be (i)-differentiable, then we have [(ii) - v]-differentiable right fuzzy Caputo derivative and

$$
\left(D_{b-}^{v} f\right)(x, r)=\left[\left(D_{b^{-}}^{v} f_{+}\right)(x, r),\left(D_{b^{-}}^{v} f_{-}\right)(x, r)\right], \quad 0 \leq r \leq 1 .
$$

Proof It is straightforward.

Theorem 3.2 Let $f \in C_{F}[a, b] \cap L_{F}[a, b], 0<v<1, a \leq x \leq b$. If $D_{b-}^{v} f(x)$ exists and it is Lebesgue integrable, then we state the equivalent integral form of the original fuzzy fractional differential equation $D_{b}^{v} f(x)=g(x, f(x))$ with the initial condition $f_{0}=f(b)$ as follows:

(i) iff is a $[(i)-v]$-differentiable fuzzy-valued function, then

$$
f(x)=f(b) \oplus \frac{1}{\Gamma(\nu)} \odot \int_{x}^{b}(t-x)^{\nu-1} \odot\left(D_{b^{-}}^{v} f\right)(t) d t .
$$

(ii) iff is a $[(i i)-v]$-differentiable fuzzy-valued function, then

$$
f(x)=f(b) \ominus \frac{-1}{\Gamma(\nu)} \odot \int_{x}^{b}(t-x)^{\nu-1} \odot\left(D_{b-}^{\nu} f\right)(t) d t .
$$

Proof [25].

Now, we state the following result which will be used later.

Theorem 3.3 Let $f \in C_{F}[a, b] \cap L_{F}[a, b]$ and $0<v<1$. Then

$$
D\left(\frac{1}{b-a} \odot(F R) \int_{a}^{b} f(x) d x, f(b)\right) \leq \frac{(b-a)^{v}}{\Gamma(v+2)} \sup _{t \in[a, b]} D\left(\left(D_{b-}^{v} f\right)(t), \tilde{0}\right) .
$$

Proof We observe that

$$
\begin{aligned}
& D\left(\frac{1}{b-a} \odot(F R) \int_{a}^{b} f(x) d x, f(b)\right) \\
& \quad=D\left(\frac{1}{b-a} \odot(F R) \int_{a}^{b} f(x) d x, \frac{f(b)}{b-a} \int_{a}^{b} d x\right)
\end{aligned}
$$




$$
\begin{aligned}
& =D\left(\frac{1}{b-a} \odot(F R) \int_{a}^{b} f(x) d x, \frac{1}{b-a} \odot(F R) \int_{a}^{b} f(b) d x\right) \\
& =\frac{1}{b-a} D\left((F R) \int_{a}^{b} f(x) d x,(F R) \int_{a}^{b} f(b) d x\right) \\
& \leq \frac{1}{b-a} \int_{a}^{b} D(f(x), f(b)) d x
\end{aligned}
$$

for the case $[(i)-v]$-differentiable. We notice that $f \in C_{F}[a, b] \cap L_{F}[a, b]$ and $0<v<1$,

$$
f(x)=f(b) \oplus \frac{1}{\Gamma(\nu)} \odot \int_{x}^{b}(t-x)^{\nu-1} \odot\left(D_{b^{-}}^{\nu} f\right)(t) d t .
$$

For $a \leq x \leq b$, we have

$$
\begin{aligned}
D(f(x), f(b)) & =D\left(f(b) \oplus \frac{1}{\Gamma(v)} \odot \int_{x}^{b}(t-x)^{\nu-1} \odot\left(D_{b^{-}}^{v} f\right)(t) d t, f(b)\right) \\
& =D\left(\frac{1}{\Gamma(v)} \odot \int_{x}^{b}(t-x)^{\nu-1} \odot\left(D_{b^{-}}^{v} f\right)(t) d t, \tilde{0}\right) \\
& \leq \frac{1}{\Gamma(v)} D\left(\int_{x}^{b}(t-x)^{\nu-1} \odot\left(D_{b^{-}}^{v} f\right)(t) d t, \tilde{0}\right) \\
& \leq \frac{1}{\Gamma(v)} D\left(\int_{x}^{b}(t-x)^{\nu-1} \odot\left(D_{b^{-}}^{v} f\right)(t) d t, \int_{x}^{b} \tilde{0} d t\right) \\
& \leq \frac{1}{\Gamma(v)} \int_{x}^{b}(t-x)^{\nu-1} D\left(\left(D_{b^{-}}^{v} f\right)(t), \tilde{0}\right) d t \\
& \leq \frac{1}{\Gamma(v)} \frac{(b-x)^{v}}{v} \sup _{t \in[a, b]} D\left(\left(D_{b^{-}}^{v} f\right)(t), \tilde{0}\right) .
\end{aligned}
$$

As a result, we prove that

$$
D(f(x), f(b)) \leq \frac{(b-x)^{v}}{\Gamma(v+1)} \sup _{t \in[a, b]} D\left(\left(D_{b}^{v} f\right)(t), \tilde{0}\right), \quad a \leq x \leq b .
$$

Thus, we obtain

$$
\int_{a}^{c} D(f(x), f(b)) d x \leq \frac{(b-a)^{v+1}}{\Gamma(v+2)} \sup _{t \in[a, b]} D\left(\left(D_{b-}^{v} f\right)(t), \tilde{0}\right) .
$$

In the case $[(i)-v]$-differentiable, we have

$$
\begin{aligned}
D\left(\frac{1}{b-a} \odot(F R) \int_{a}^{b} f(x) d x, f(b)\right) & \leq \frac{1}{b-a} \int_{a}^{b} D(f(x), f(b)) d x \\
& \leq \frac{(b-a)^{v}}{\Gamma(v+2)} \sup _{t \in[a, b]} D\left(\left(D_{b}^{v}-f\right)(t), \tilde{0}\right)
\end{aligned}
$$

for the case $[(i i)-v]$-differentiable. We notice that $f \in C_{F}[a, b] \cap L_{F}[a, b]$ and $0<v<1$, also

$$
f(x)=f(b) \ominus \frac{-1}{\Gamma(\nu)} \odot \int_{x}^{b}(t-x)^{\nu-1} \odot\left(D_{b-}^{v} f\right)(t) d t .
$$


Thus, for all $a \leq x \leq b$, we have

$$
\begin{aligned}
D(f(x), f(b)) & =D\left(f(b) \ominus \frac{-1}{\Gamma(v)} \odot \int_{x}^{b}(t-x)^{\nu-1} \odot\left(D_{b^{-}}^{v} f\right)(t) d t, f(b)\right) \\
& =D\left(\frac{1}{\Gamma(v)} \odot \int_{x}^{b}(t-x)^{\nu-1} \odot\left(D_{b^{-}}^{v} f\right)(t) d t, \tilde{0}\right) \\
& \leq \frac{1}{\Gamma(v)} D\left(\int_{x}^{b}(t-x)^{\nu-1} \odot\left(D_{b^{-}}^{v} f\right)(t) d t, \tilde{0}\right) \\
& \leq \frac{1}{\Gamma(v)} D\left(\int_{x}^{b}(t-x)^{\nu-1} \odot\left(D_{b^{-}}^{v} f\right)(t) d t, \int_{x}^{b} \tilde{0} d t\right) \\
& \leq \frac{1}{\Gamma(v)} \int_{x}^{b}(t-x)^{\nu-1} D\left(\left(D_{b^{-}}^{v} f\right)(t), \tilde{0}\right) d t \\
& \leq \frac{1}{\Gamma(v)} \frac{(b-x)^{v}}{v} \sup _{t \in[a, b]} D\left(\left(D_{b^{-}}^{v} f\right)(t), \tilde{0}\right) .
\end{aligned}
$$

So, we prove that

$$
D(f(x), f(b)) \leq \frac{(b-x)^{v}}{\Gamma(v+1)} \sup _{t \in[a, b]} D\left(\left(D_{b^{-}}^{v} f\right)(t), \tilde{0}\right), \quad a \leq x \leq b
$$

Thus, we obtain

$$
\int_{a}^{c} D(f(x), f(b)) d x \leq \frac{(b-a)^{v+1}}{\Gamma(v+2)} \sup _{t \in[a, b]} D\left(\left(D_{b^{-}}^{v} f\right)(t), \tilde{0}\right) .
$$

When $[(i i)-v]$-differentiable, we have

$$
\begin{aligned}
D\left(\frac{1}{b-a} \odot(F R) \int_{a}^{b} f(x) d x, f(b)\right) & \leq \frac{1}{b-a} \int_{a}^{b} D(f(x), f(b)) d x \\
& \leq \frac{(b-a)^{v}}{\Gamma(v+2)} \sup _{t \in[a, b]} D\left(\left(D_{b^{-}}^{v} f\right)(t), \tilde{0}\right) .
\end{aligned}
$$

This completes the proof.

Now, we state the main result as given below.

Theorem 3.4 Let $f, g, h \in C_{F}[a, b] \cap L_{F}[a, b]$ and $0<v<1$. Then

$$
\begin{aligned}
& D\left(3 \int_{a}^{b} f(x) g(x) h(x) d x, \int_{a}^{b}[f(b) g(x) h(x)+f(x) g(b) h(x)+f(x) g(x) h(b)] d x\right) \\
& \leq I_{a+}^{v+1}(h(b) g(b)) \sup _{t \in[a, b]} D\left(\left(D_{b^{-}}^{v} f\right)(t), \tilde{0}\right) \\
& \quad+I_{a+}^{v+1}(f(b) h(b)) \sup _{t \in[a, b]} D\left(\left(D_{b^{-}}^{v} g\right)(t), \tilde{0}\right) \\
& \quad+I_{a+}^{v+1}(f(b) g(b)) \sup _{t \in[a, b]} D\left(\left(D_{b^{-}}^{v} h\right)(t), \tilde{0}\right) .
\end{aligned}
$$


Proof For $f, g, h \in C_{F}[a, b] \cap L_{F}[a, b]$ we have

$$
\begin{gathered}
D\left(3 \int_{a}^{b} f(x) g(x) h(x) d x, \int_{a}^{b}[f(b) g(x) h(x)+f(x) g(b) h(x)+f(x) g(x) h(b)] d x\right) \\
=\int_{a}^{b}(D(3 f(x) g(x) h(x),[f(b) g(x) h(x)+f(x) g(b) h(x)+f(x) g(x) h(b)])) d x .
\end{gathered}
$$

So we have the following:

$$
\begin{aligned}
& \int_{a}^{b}(D(3 f(x) g(x) h(x),[f(b) g(x) h(x)+f(x) g(b) h(x)+f(x) g(x) h(b)])) d x \\
& =\int_{a}^{b}[D(f(x) g(x) h(x)+f(x) g(x) h(x)+f(x) g(x) h(x), \\
& [f(b) g(x) h(x)+f(x) g(b) h(x)+f(x) g(x) h(b)])] d x \\
& =\int_{a}^{b}[D(f(x) g(x) h(x), f(b) g(x) h(x))+D(f(x) g(x) h(x), f(x) g(b) h(x)) \\
& +D(f(x) g(x) h(x), f(x) g(x) h(b))] d x \\
& =\int_{a}^{b}[g(x) h(x) D(f(x), f(b))+f(x) h(x) D(g(x), g(b))+f(x) g(x) D(h(x), h(b))] d x \\
& =\int_{a}^{b}\left[g(x) h(x)\left(\frac{(b-x)^{v}}{\Gamma(v+1)} \sup _{t \in[a, b]} D\left(\left(D_{b}^{v}-f\right)(t), \tilde{0}\right)\right)\right. \\
& +f(x) h(x)\left(\frac{(b-x)^{v}}{\Gamma(v+1)} \sup _{t \in[a, b]} D\left(\left(D_{b^{-}}^{v} g\right)(t), \tilde{0}\right)\right) \\
& \left.+f(x) g(x)\left(\frac{(b-x)^{v}}{\Gamma(v+1)} \sup _{t \in[a, b]} D\left(\left(D_{b^{-}}^{v} h\right)(t), \tilde{0}\right)\right)\right] d x \\
& \leq \int_{a}^{b}\left[g(x) h(x)\left(\frac{(b-x)^{v}}{\Gamma(v+1)} \sup _{t \in[a, b]} D\left(\left(D_{b-}^{v} f\right)(t), \tilde{0}\right)\right)\right] d x \\
& +\int_{a}^{b}\left[f(x) h(x)\left(\frac{(b-x)^{v}}{\Gamma(v+1)} \sup _{t \in[a, b]} D\left(\left(D_{b^{-}}^{v} g\right)(t), \tilde{0}\right)\right)\right] d x \\
& +\int_{a}^{b}\left[f(x) g(x)\left(\frac{(b-x)^{v}}{\Gamma(v+1)} \sup _{t \in[a, b]} D\left(\left(D_{b^{-}}^{v} h\right)(t), \tilde{0}\right)\right)\right] d x \\
& \leq \sup _{t \in[a, b]} D\left(\left(D_{b^{-}}^{\nu} f\right)(t), \tilde{0}\right)\left(\frac{1}{\Gamma(v+1)} \int_{a}^{b}\left[g(x) h(x)(b-x)^{\nu}\right] d x\right) \\
& +\sup _{t \in[a, b]} D\left(\left(D_{b^{-}}^{v} g\right)(t), \tilde{0}\right)\left(\frac{1}{\Gamma(v+1)} \int_{a}^{b}\left[f(x) h(x)(b-x)^{\nu}\right] d x\right) \\
& +\sup _{t \in[a, b]} D\left(\left(D_{b^{-}}^{v} h\right)(t), \tilde{0}\right)\left(\frac{1}{\Gamma(v+1)} \int_{a}^{b}\left[f(x) g(x)(b-x)^{\nu}\right] d x\right) \\
& =I_{a+}^{v+1}(h(b) g(b)) \sup _{t \in[a, b]} D\left(\left(D_{b^{-}}^{v} f\right)(t), \tilde{0}\right)+I_{a+}^{v+1}(f(b) h(b)) \sup _{t \in[a, b]} D\left(\left(D_{b^{-}}^{v} g\right)(t), \tilde{0}\right) \\
& +I_{a+}^{v+1}(f(b) g(b)) \sup _{t \in[a, b]} D\left(\left(D_{b^{-}}^{v} h\right)(t), \tilde{0}\right) .
\end{aligned}
$$


Finally, by using the following property:

$$
I_{a+}^{v+1}(h(b) g(b))=\frac{1}{\Gamma(v+1)} \int_{a}^{b}\left[g(x) h(x)(b-x)^{v}\right] d x,
$$

we complete the proof.

\section{Conclusion}

Recently, the application of fractional differential equations under uncertainty received a considerable interest both in mathematics and in applications. In this manuscript, the fuzzy Caputo differentiability has been stated; then we made inquiries about the fuzzy fractional Ostrowski inequality involving three functions in the right Caputo fractional derivative. In this way, we have obtained some basic results in the fuzzy framework. To the best of our knowledge, this is the first time in the literature that such inequality has been considered under uncertainty. For future work, we will consider the mentioned fuzzy inequality possessing higher fractional order with different types of differentiability.

\section{Competing interests}

The authors declare that they have no competing interests.

Authors' contributions

All authors have equal contributions.

\section{Author details}

${ }^{1}$ Department of Mathematics, Science and Research Branch, Islamic Azad University, Tehran, Iran. ${ }^{2}$ Department of Mathematics, Yasooj Branch, Islamic Azad University, Yasooj, Iran. ${ }^{3}$ Faculty of Marine Science, Chabahar Maritime University, Chabahar, Iran. ${ }^{4}$ Department of Mathematics and Computer Sciences, Cankaya University, Ankara, 06530, Turkey. ${ }^{5}$ Department of Chemical and Materials Engineering, Faculty of Engineering, King Abdulaziz University, Jeddah, Saudi Arabia. ${ }^{6}$ Institute of Space Sciences, Magurele, Bucharest, Romania. ${ }^{7}$ Young Researchers and Elite Club, Mobarakeh Branch, Islamic Azad University, Mobarakeh, Iran.

\section{Acknowledgements}

The reviewers' comments, which have improved the quality of this paper, are greatly appreciated.

Received: 25 November 2012 Accepted: 24 January 2013 Published: 14 February 2013

\section{References}

1. Ostrowski, A: Über die absolutabweichung einer differentiebaren funktion von ihrem intetegralmittelwert. Comment. Math. Helv. 10, 226-227 (1938)

2. Milovanovif, GV, Pecaric, JE: On generalization of the inequality of A. Ostrowski and some related applications. Univ. Beograd. Publ. Elektrotehn. Fak. Ser. Mat. Fiz. 44, 155-158 (1976)

3. Pachpatte, BG: On an inequality of Ostrowski type in three independent variables. J. Math. Anal. Appl. 249, 583-591 (2000)

4. Pachpatte, BG: On a new Ostrowski type inequality in two independent variables. Tamkang J. Math. 32, 45-49 (2001)

5. Rafiq, A, Mir, NA, Ahmad, F: Weighted Chebyshev-Ostrowski type inequalities. Appl. Math. Mech. 28, $901-906$ (2007)

6. Sarikaya, MZ: On the Ostrowski type integral inequality. Acta Math. Univ. Comen. 1, 129-134 (2010)

7. Ujevic, N: Sharp inequalities of Simpson type and Ostrowski type. Comput. Math. Appl. 48, 145-151 (2004)

8. Zhongxue, L: On sharp inequalities of Simpson type and Ostrowski type in two independent variables. Comput. Math. Appl. 56, 2043-2047 (2008)

9. Alomari, M, Darus, M: Some Ostrowski type inequalities for convex functions with applications. RGMIA 13 (2010, preprint)

10. Alomari, M, Darus, M, Dragomir, S, Cerone, P: Ostrowski type inequalities for functions whose derivatives are $s$-convex in the second sense. Appl. Math. Lett. 23, 1071-1076 (2010)

11. Dragomir, SS, Wang, S: An inequality of Ostrowski-Gruss type and its applications to the estimation of error bounds for some special means and for some numerical quadrature rules. Comput. Math. Appl. 33, 15-20 (1997)

12. Matic, M, Plaric, J, Ujveif, N: Improvement and further generalization of some inequalities of Ostrowski-Griiss type. Comput. Math. Appl. 39, 161-175 (2000)

13. Cheng, XL: Improvement of some Ostrowski-Gruss type inequalities. Comput. Math. Appl. 42, 109-114 (2001)

14. Pachpatte, BG: New Ostrowski and Gruss type inequalities. An. Ştiinţ. Univ. "Al. I. Cuza" laşi, Mat. LI(2), $377-386$ (2005)

15. Anastassiou, GA: Fuzzy Ostrowski inequalities. In: Fuzzy Mathematics: Approximation Theory, vol. 251, pp. 65-73. Springer, Berlin (2010)

16. Anastassiou, GA: On right fractional calculus. Chaos Solitons Fractals 42, 365-376 (2009) 
17. Bohner, EA, Bohner, M, Mattews, T: Time scales Ostrowski and Gruss type inequalities involving three functions Nonlinear Dyn. Syst. Theory 8, 651-700 (2011)

18. Bohner, M, Mattews, T: Ostrowski inequalities on time scales. J. Inequal. Pure Appl. Math. 9, 6-8 (2008)

19. Tseng, KL: Improvement of some inequalities of Ostrowski type and their application. Taiwan. J. Math. 12, 2427-2441 (2008)

20. Dragomir, SS, Wang, S: A new equality of Ostrowski's type in $L_{1}$ norm and applications to some special means and to some numerical quadrature rules. Tamkang J. Math. 28, 239-244 (1997)

21. Allahviranloo, T, Salahshour, S: A new approach for solving first order fuzzy differential equation. In: Inf. Proc. Manag. Uncert. Know. Bas. Syst. Appl. (2010)

22. Allahviranloo, T, Salahshour, S: Euler method for solving hybrid fuzzy differential equation. Soft Comput. Fus. Found. Meth. Appl. 7, 1247-1253 (2010)

23. Allahviranloo, T, Salahshour, S, Abbasbandy, S: Explicit solutions of fractional differential equations with uncertainty. Soft Comput. Fus. Found. Meth. Appl. 16, 297-302 (2012)

24. Salahshour, S, Allahviranloo, T, Abbasbandy, S, Baleanu, D: Existence and uniqueness results for fractional differential equations with uncertainty. Adv. Differ. Equ. 112, 1-13 (2012)

25. Allahviranloo, T, Salahshour, S, Avazpour, L: On the fractional Ostrowski inequality with uncertainty. J. Math. Anal. Appl. 395, 191-201 (2012)

doi:10.1186/1029-242X-2013-50

Cite this article as: Allahviranloo et al.: Retracted: Fuzzy fractional Ostrowski inequality with Caputo differentiability. Journal of Inequalities and Applications 2013 2013:50.

Submit your manuscript to a SpringerOpen ${ }^{\circ}$ journal and benefit from:

- Convenient online submission

- Rigorous peer review

- Immediate publication on acceptance

- Open access: articles freely available online

- High visibility within the field

- Retaining the copyright to your article

Submit your next manuscript at $>$ springeropen.com 